2017-08-01

\title{
RANS-VOF modelling of the Wavestar point absorber
}

\author{
Ransley, E::0000-0003-1446-7907
}

http://hdl.handle.net/10026.1/8586

10.1016/j.renene.2017.02.079

Renewable Energy

Elsevier BV

All content in PEARL is protected by copyright law. Author manuscripts are made available in accordance with publisher policies. Please cite only the published version using the details provided on the item record or document. In the absence of an open licence (e.g. Creative Commons), permissions for further reuse of content should be sought from the publisher or author. 


\title{
RANS-VOF Modelling of the Wavestar Point Absorber
}

\author{
E. J. Ransley ${ }^{\mathrm{a}, *}$, D. M. Greaves ${ }^{\mathrm{a}}$, A. Raby ${ }^{\mathrm{a}}$, D. Simmonds ${ }^{\mathrm{a}}$, M. M. Jakobsen ${ }^{\mathrm{b}}$, \\ M. Kramer ${ }^{\mathrm{b}}$ \\ ${ }^{a}$ School of Marine Science and Engineering, Plymouth University, UK \\ ${ }^{b}$ Department of Civil Engineering, Aalborg University, Denmark
}

\begin{abstract}
Numerical modelling has become commonplace in the development of offshore structures, however for dynamic systems such as Wave Energy Convertors (WECs) conventional numerical tools may not be able to capture the full range of behaviours required for engineering analysis. This is particularly problematic in extreme seas where the nonlinearities and coupled nature of these systems become important. In this work a fully nonlinear, coupled tool for simulation of WECs is produced and compare with physical measurements. Regular wave interactions with both a fixed and freely-pitching, 1:10 scale model of the Wavestar are reproduced numerically. The numerical model is shown to be capable of predicting the pressure on the float's surface and the fully coupled motion of the device. However, the results indicate that the higher-order free surface behaviour in the vicinity of the device is not being captured correctly. Finally, the numerical model is shown to cope with an extreme regular wave including large amplitude motion, full submersion and high levels of free surface distortion. The results presented suggest that the quality of the numerical reproduction does not decrease with wave steepness; however the execution time of simulations increases significantly with increased float oscillation.
\end{abstract}

Keywords: Wave Energy Converter (WEC), Computational Fluid Dynamics (CFD), OpenFOAM, coupled, time domain, regular waves 2015 MSC: 00-01, 99-00

\footnotetext{
${ }^{*}$ Corresponding author Email address: edward.ransley@plymouth.ac.uk (E. J. Ransley)
}

Preprint submitted to Renewable Energy

February 7, 2017 


\section{Introduction}

The continued increase in the performance-to-cost ratio of modern computers has meant numerical models can now provide the quantitative description required for the engineering analysis of marine structures, like Wave Energy

5 Converters (WECs) without the need for an exhaustive series of complex, and sometimes expensive, physical experiments [1]. Furthermore, numerical simulations offer a means to interpret the fundamental phenomenological aspects of experimental conditions at full-scale that physical tests may not. As a result, the 'Review of model testing requirements for FPSOs' 2] (referred to in the 'Guidelines on design and operation of wave energy converters' $[3$ for issues related to wave structure model testing) states that optimisation of both the vessel and mooring system should typically now be performed on a computer, while physical model testing should be reserved for purposes of validation and design confirmation. This is supported by the Technology Readiness Levels (TRLs) which: promote the use of linear frequency-domain models, like Morison's load formula [4, and hydrodynamic inputs from potential flow solvers, like WAMIT [5], to assess various concepts in TRL 3 ; recommend time-domain models, such as the ANSYS AQWA Suite [6] or mooring system design software Orcaflex [7, to formulate a single, advance concept design in TRL 4; and suggest fully nonlinear models such as Navier-Stokes (NS) solvers and Computational Fluid Dynamics (CFD) methods for fluid-structure nonlinearities 'if necessary' and direct coupling between the various component models in TRL 5 [8].

Despite these recommendations, numerical simulations can be extremely time consuming without proper implementation and it is possible for a quantitatively incorrect solution to look reasonable. Without validation from physical tests, the consequences of accepting such a result may be severe [9] and so, numerical models do not provide a substitute to physical experiments; the two form a pair of complementary development tools. Furthermore, unlike conventional offshore structures, WECs are designed to develop specific motions under 
wave loading. So, whilst a WEC system must constrain or withstand unwanted motions that may cause damage to the device, it must also be responsive to those motions which produce power. It is therefore unclear as to how much of the existing research is directly transferable to cases involving dynamically responsive WECs and essential that additional work is done to update the formal guidelines to reflect the differences between WECS and other offshore structures.

Therefore, the aim of this work is to produce and validate a fully nonlinear, coupled model of an existing $\mathrm{WEC}$ and use it to assess the performance of such models when considering dynamically responsive structures such of WECs.

In this article, the particular WEC under investigation, the Wavestar, is in40 troduced along with a description of the physical validation experiments. Similar work from the literature is reviewed and then the design of the Numerical Wave Tank (NWT) is described. A pair of regular waves with different steepnesses are generated numerically and results are presented for both a fixed float and one able to move freely in pitch.

\section{The Wavestar Machine}

First proposed in 2000 by Niels and Keld Hansen, the Wavestar concept has become one of the world-leading wave energy technologies [10, boasting one of the very few full-scale, grid-connected prototypes to undergo real sea trials. The Wavestar machine is a point-absorber-type WEC which generates electricity through the oscillatory motion of hemispherical floats (Figure 17a) which in turn drive hydraulic Power Take-Off (PTO) systems. Each float is connected to a static super-structure which is held stationary via a number of monopile-type foundations (Figure $1 \mathrm{~b}$ ). This constrains the motion of the floats to a single rotational degree of freedom about the hinge point between the support structure and the float arm.

Over the previous decade, a range of scale experiments and real sea tests have been performed in order to investigate different aspects of the device design [10, 11, 12. In a recent set of experiments, performed in the COAST Lab- 

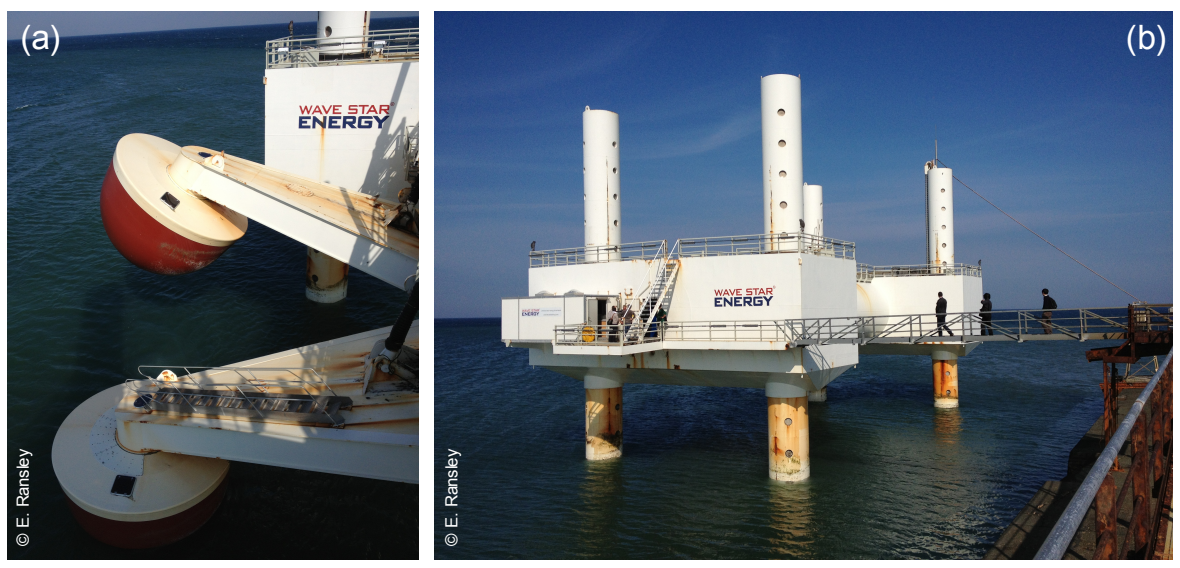

Figure 1: Photographs of the Wavestar prototype near Hanstholm, on the west coast of Denmark. Showing 2 point-absorbing floaters (a) and the supporting platform and 4 monopile foundations (b). Photo Credit: E. Ransley (Sept. 2013).

oratory Ocean basin at Plymouth University and supported by MARINET, the survivability of the device under extreme conditions was assessed in a controlled environment [13. A 1:10 scale model of a single float and arm was mounted on the gantry in the basin using a custom-fitted frame and a set of ball-bearings to allow the system to pitch. In the experiment, a hydraulic piston, with variable damping and stiffness, was used to simulate the PTO of the full scale device.

${ }_{65}$ Figure 2 shows the experimental set-up and the array of sensors used to measure the force on the float, the pressure exerted on the surface of the float, the surface elevation in the vicinity of the float and the particle velocities near the float. A series of experiments were performed including regular wave tests of varied steepness with the float either fixed in place or allowed to move freely with the hydraulic system disabled, i.e. no PTO damping. In this article, a subset of these regular wave experiments has been produced using the NWT developed by Ransley [14] and compared with the experimental measurements. In the interest of promoting an incremental development strategy for the NWT, cases with the PTO engaged have not been considered in this work. An as75 sessment of the quality of the NWT as a function of incident wave steepness, 

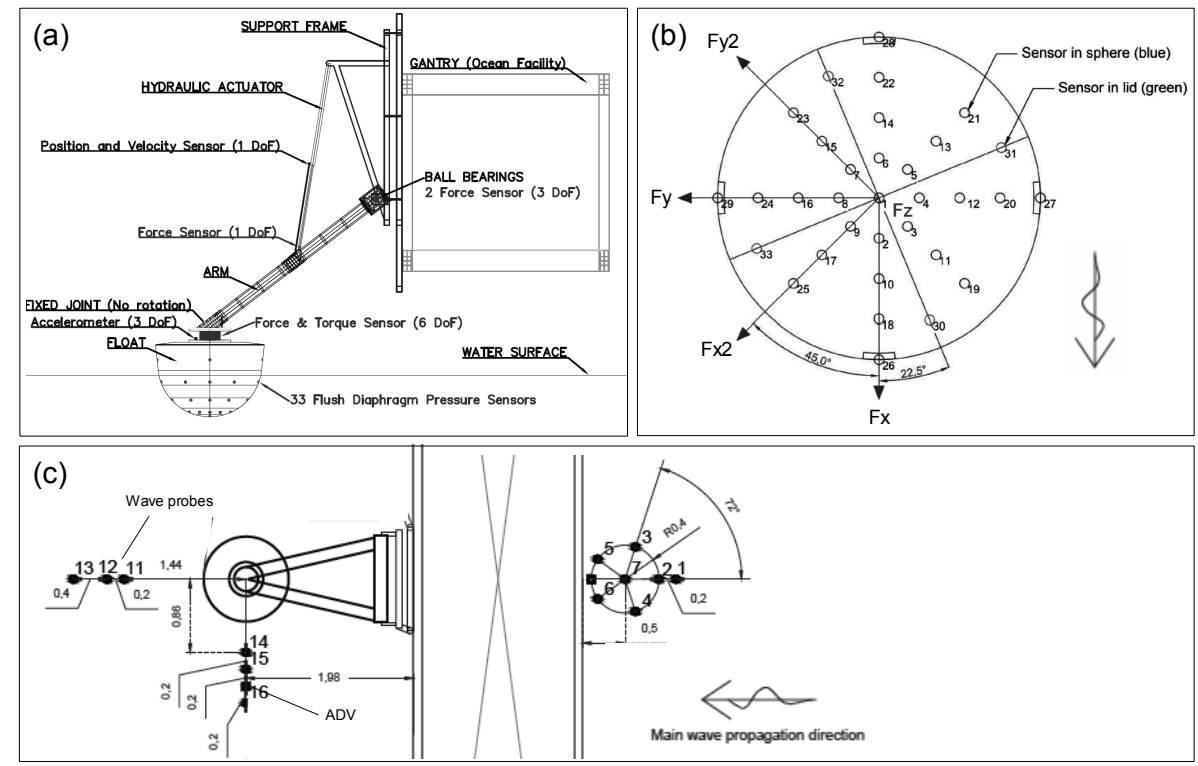

Figure 2: Schematics of the Wavestar experimental setup. (a) Profile of the model, frame and gantry-mounting showing the position of the force and torque sensors. (b) Plan view of the float showing the positions of the pressure sensors ( $\mathrm{Fx}$ is the incident wave direction). (c) Plan view of the physical test area showing the positions of the wave gauges and velocimeters.

has then been made along with a discussion on the ability of fully nonlinear CFD methods to model the coupled motion of WECs. A full description of the complete experimental program is given by Jakobsen [15].

\section{Literature}

In the case of nonlinear, time-domain simulations including multiphase flows and dynamic systems such as wave energy devices, it is common for the number of degrees of freedom to be artificially restricted. This makes the application and analysis of their behaviour easier to interpret. For the Wavestar, however, the structure is only able to move in a single degree of freedom (pitch), provid85 ing the necessary gradual increase in complexity from fixed structures without simplifying the actual behaviour of the device. Furthermore, the restriction on 
the degrees of freedom provides all of the required constraints on the body's motion without the need for additional restraints such as moorings.

In much of the relevant literature the complexity of the model is reduced even further by 'driving' the motion of the structure and therefore removing any coupling between the pressure and viscous forces from the fluid and the movement of the structure.

Qian et al. [16 modelled the entry and total immersion of a wedge-shaped body driven with constant vertical velocity into initially calm water. In a separate simulation they also modelled the emergence of the wedge from beneath the water's surface. Their fully nonlinear NS method was based on the Cartesian cut cell technique for tracking moving solid boundaries and, despite having no experimental data for comparison, they showed it was capable of handling complex two-phase flows with moving bodies, interface break-up and recombination.

Kleefsman et al. 17] present results for constant velocity water entry of two 2D wedges and a 3D cone using the Volume Of Fluid (VOF) based NS solver ComFLOW. They found that, with a fine grid, ComFLOW was able to resolve the jets on either side of the wedge in good agreement with visual observations. However, not all of the droplets and smaller details were captured by the model. In 3D, and with a coarser grid, the jets on the side of a 3D cone were less well resolved but the slamming coefficient was found to be in good agreement with theory. Kleefsman et al. 17] also showed a comparison between numerical simulations and experimental results for the entry of a circular cylinder with constant velocity. They found that the free surface shape and the vertical hydrodynamic force computed compared well with experiments but the initial impact was slightly underestimated by ComFLOW. It was also concluded that a very fine mesh was required to resolve the free surface behaviour but this did not have a large effect on the total hydrodynamic force.

Zhang et al. [18 modelled both the entry and exit of a circular cylinder with constant velocity using their level-set immersed boundary method and a full $\mathrm{NS}$ solver. They too found good visual agreement with experiments for the free 
surface and comparable values for the slamming coefficients.

Westphalen et al. 19] solved the fully nonlinear NS equations for a cone, Gaussian wave packet. They found their control-volume Finite Element (CVFE) method predicted the high order force components on the structure well, although the model over-estimated the peak and under-estimated the minimum forces on the cone [20]. It was also found that the maximum and minimum surface elevations produced by the cone were over- and under-predicted respectively, particularly at high driving frequencies. Further-still, although the motion of the cone was not coupled with the fluid forcing, the inertia and drag forces on the structure appear to be much more complex than those for standard water entry problems i.e. there was a phase difference between the maximum flow velocities and the speed of the cone [20].

Bangun and Utsunomiya 21] investigated the viscous forces acting on a 2D barge with prescribed sinusoidal roll motion using an NS solver based on the Finite Volume Method (FVM) and using the Semi-Implicit for Pressure Link Equations (SIMPLE) algorithm. They found that viscosity plays an important 35 role in problems involving floating bodies particularly in terms of the motion damping coefficients.

As well as greatly increasing the complexity of the geometry over similar examples in the literature, in the application reported here the motion of the device is coupled to the hydrodynamic loading of the surrounding fluid. Simulations in which the motion of a moving object is calculated from the interaction between the object and the fluid dynamics are far more scarce.

Kleefsman et al. [17] modelled a wedge freely falling into initially still water using ComFLOW. When compared with experimental data they found that the force exerted on the wedge was over-predicted and consequently the vertical velocity was under-predicted.

Zhang et al. [18, also modelled the free falling wedge using their level-set method. They too found that the force was over-predicted leading to a greater deceleration and slower motion. Both Kleefsman et al. [17] and Zhang et al. [18] 
explain these discrepancies in terms of 3D effects which were not accounted for libraries with experiments of regular wave interactions with a $2 \mathrm{D}$ rectangular barge constrained to move in roll only. They found their model was able to predict the roll motion accurately but saw a large discrepancy in the damping coefficients due to the 2D flow assumptions used. Similarly to Bangun and 175 role in the rotational dynamics of floating bodies. 


\section{The Numerical Wave Tank (NWT)}

The NWT used here has been designed based on the work of Ransley [14. Using the open-source $\overline{C F D}$ libraries OpenFOAM ${ }^{\circledR}$ (version 2.3.0), the NWT solves the RANS equations for two incompressible, isothermal, immiscible fluids using a VOF-based interface capturing scheme similar to that of Ubbink [26]. Utilising the two-fluid Eulerian model, where the phase fraction equations for each fluid are solved separately, the interface-capturing method, described by Rusche [27, does not use a compressive differencing scheme. Instead an artificial compression term is added to the volume fraction equation in order to provide a sharper interface resolution [28. The boundedness of the volume fraction equa„tion is then achieved via a specially designed solver called Multi-Dimensional Limiter for Explicit Solution (MULES) [27, 29].

A segregated, iterative technique is used to solve separate matrix equations, constructed using the FVM applied to an unstructured grid, where the solution variables are co-located at the cell centres. The pressure-velocity coupling is achieved using adapted versions of either the PISO or SIMPLE algorithms which incorporate the phase fraction equation to derive a new pressure equation [30].

By including the additional toolbox waves2Foam [31, 32, regular wave generation on the inlet boundary has been achieved using a set of expression-based boundary conditions based on second-order Stokes theory. Wave absorption has been added using the passive Relaxation Zone (RZ) formulation with an exponential decay function as the weighting factor and a target solution of still water.

The computational domain was made $16 \mathrm{~m}$ long and $6 \mathrm{~m}$ wide with relaxation zones of $1 \mathrm{~m}$ and $6 \mathrm{~m}$ positioned on the inlet and outlet boundaries respectively. The water depth in each of the experiments was $3 \mathrm{~m}$ and the height of the air phase was $3.75 \mathrm{~m}$ to accommodate the device arm and allow sufficient space for the mesh to deform. The background mesh was made up of cubic cells with edge length $0.2 \mathrm{~m}$. The region containing the free surface was then refined by two levels using an octree refinement strategy giving a cell edge length of $0.05 \mathrm{~m}$. 

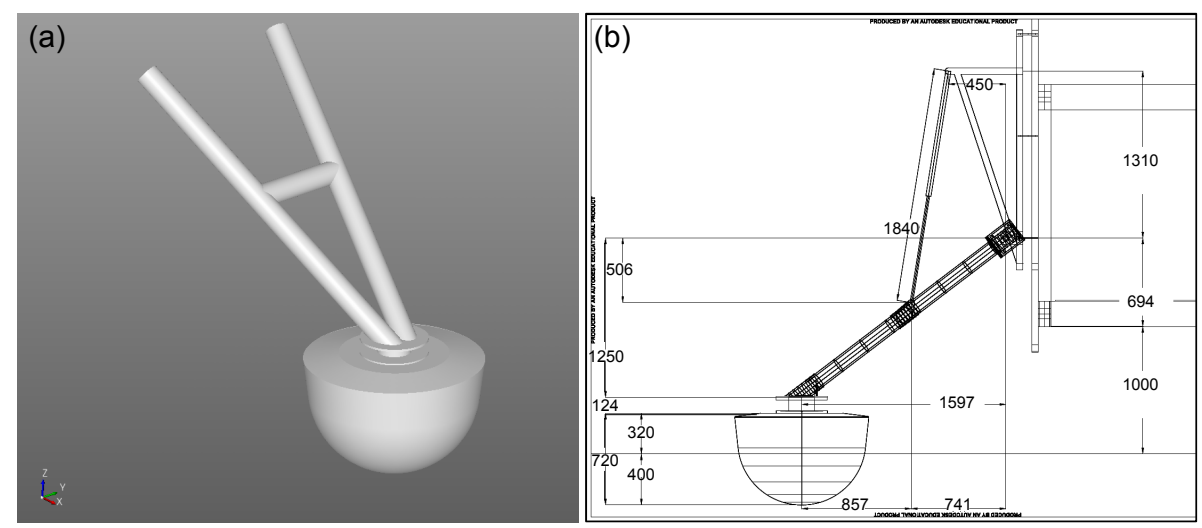

Figure 3: (a) Oblique view of the Wavestar CAD model, produced using Salome 7.2. (b) Dimensions of the 1:10 scale model of the Wavestar machine

Based on the parametric study made by Ransley [14, this was considered to be sufficient for mesh independence, in the two wave cases considered here, with a Root Mean Squared (RMS) error of $2 \%$. A Computer-Aided Design (CAD) model with the same dimensions as the physical model was produced (Figure 3 ) and the region occupied by the structure was extracted from the mesh using the snappyHexMesh utility. The middle of the float was positioned centrally widthways, $4.97 \mathrm{~m}$ from the inlet and the boundary of the structure was allowed a minimum refinement of level 2 or a maximum of level 3 when the cell being refined had multiple intersections with the structure surface. Figure 4 shows a cross section of the mesh.

OpenFOAM ${ }^{\circledR}$ is distributed with a range of dynamic mesh functionality including a Six Degrees Of Freedom (6DOF) rigid body motion solver. This solver is specified via a moving wall velocity condition on the patch describing the boundary of the body and has been used here to include the coupled motion of the device. Automatic mesh motion is achieved via a force calculation on this patch and the mass properties of the structure. The position of grid nodes coinciding with the surface of the structure are then updated accordingly at every timestep. In this case, the mass of the scale model device was initially 


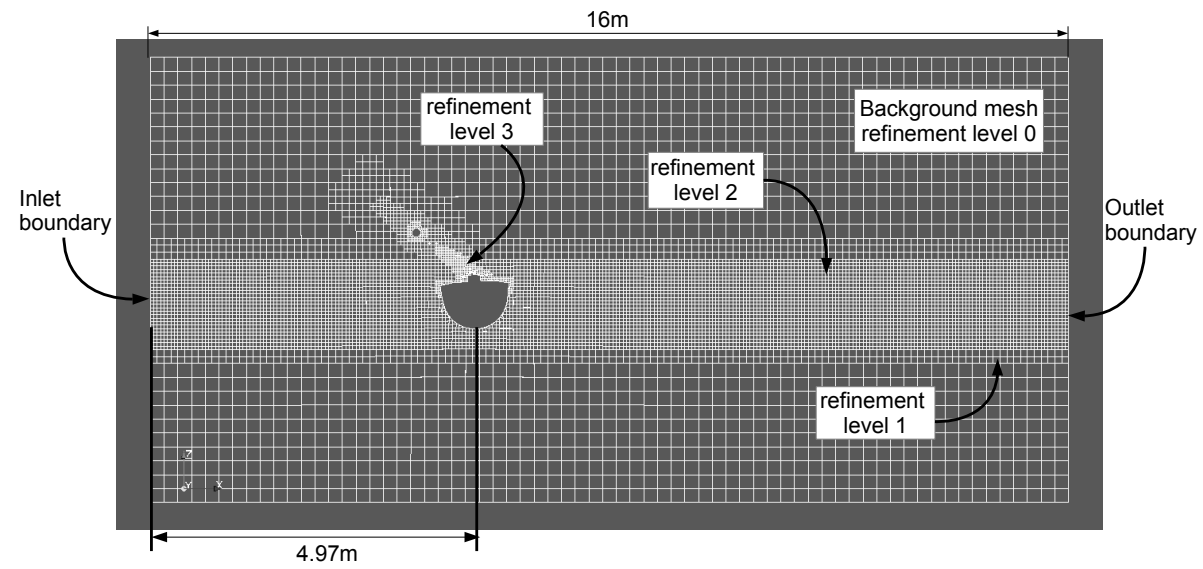

Figure 4: Cross-section of the mesh used in the Wavestar simulations at $\mathrm{t}=0 \mathrm{~s}$.

centred at (1.3954 $0.0-1.3305) \mathrm{m}$ relative to the hinge point of the arm with a magnitude of $220 \mathrm{~kg}$; the moment of inertia for pitch motion was estimated as $124.26 \mathrm{~kg} \mathrm{~m}^{2}$ using the parallel axis theorem and the mass distribution of the individual parts of the structure (this was later corroborated by results from free oscillatory tests). The motion in this case is unique and in order to model the behaviour of the Wavestar machine accurately, a pair of constraints were implemented using an explicit correction to the motion solution [30. Firstly, the device was constrained to rotate about an axis passing through the positions of the two bearings on the gantry end of the device arm. Then, a point constraint was added to this axis to remove any heave, surge or sway motion. In order to limit the degradation of mesh quality near the body and reduce the shearing of mesh cells, a region close to the structure, with a radial distance parameter of $0.1 \mathrm{~m}$, was set to remain static relative to the structure. A further radial distance parameter of $3 \mathrm{~m}$ was used to limit the extent of the deforming region in which grid node positions are updated based on spherical linear interpolation with a cosine profile in the distancing function [30.

Despite this, simulations with rigid body motion can easily become unstable when there are rapid changes in the velocity of the structure over a 
timestep. Fluctuations in the body's acceleration can occur in some cases due to 'over-shoots', followed by corrective 'under-shoots', in the calculated velocity of the structure. It has been found that the simulation is particularly sensitive to this issue when the timestep becomes very small, i.e. when small discrepancies in the velocity can lead to very large discrepancies in the acceleration. In order to resolve this issue, the acceleration of the structure can be restricted between timesteps using the accelerationRelaxation factor in the rigid body solver, where a value of 1 corresponds to no relaxation of the acceleration and a value of 0 stops any change in velocity altogether. An accelerationRelaxation of 0.7 was found to be sufficient to stabilise the most extreme cases here and so was used throughout the investigation. It is believed that an excessive accelerationRelaxation would artificially damp the motion of the structure by enforcing an underestimation of the acceleration at every timestep. However, assessment of any damping of the motion caused by the accelerationRelaxation used here remains a task for future studies.

In this work the pressure and phase fraction boundary conditions on all moving structures were the fixedFluxPressure condition and a zero gradient condition respectively.

\section{Results and Discussion}

Test conditions comprise a pair of regular wave cases with the Wavestar device both fixed in place and able to freely pitch about the hinge point of the device. Details of the two regular waves are summarised in Table 1 .

Table 1: Summary of regular wave characteristics used

\begin{tabular}{|c|c|c|c|}
\hline Wave & height, $H(\mathbf{m})$ & period, $T(\mathbf{s})$ & steepness $(H / \lambda)$ \\
\hline \hline A & 0.25 & 2.8 & 0.022 \\
\hline B & 0.15 & 1.4 & 0.049 \\
\hline
\end{tabular}

The surface elevation and fluid velocity at the inlet were prescribed using waves2Foam's second-order Stokes theory expression-based boundary condi- 
tions. For the fixed cases, numerical wave and pressure gauges were positioned at locations corresponding to those in the physical experiments and the force and moment about the position of the physical force and torque sensor (just above the float) was also recorded at every timestep. In the freely-pitching cases the position of the device's Centre of Mass (CoM) was recorded at every timestep and the pressure distribution over the float's surface was outputted at discrete times of interest throughout the wave cycle.

\subsection{Fixed cases}

For the fixed cases the physical device was locked in place in its neutrallybuoyant position whilst in the numerical simulations the mesh motion associated with the device's movement was deactivated.

Figures 5 and 6 show a sample of the surface elevation results from the numerical simulations compared to measurements taken in the two corresponding physical experiments. The line numbers correspond to the wave gauge labels in Figure 2, i.e. running from (a)-(e): 2 is positioned close to the wave maker ( $4.77 \mathrm{~m}$ upstream of the float centre), 11 and 13 are downstream of the float centre by $1.44 \mathrm{~m}$ and $2.04 \mathrm{~m}$ respectively, and 14 and 15 are $0.86 \mathrm{~m}$ and $1.46 \mathrm{~m}$ along-crest of the float centre respectively.

Plot (a) in each figure shows how well the incident waves have been reproduced. Clearly in the second (steeper) case, B, (Figure 6) the incident wave has been reproduced very well with only minor crest over-estimations. This provides confidence in the parametric mesh design which in this case only has 3 mesh cells over the height of the wave. For case A (Figure 5) the numerical reproduction is not as good, the crest heights are consistently larger than in the physical case. It is possible that there has been some error in the zero offset of the physical records, although, the noise on the crests indicates some possible vibration of the physical wave gauge. This may be as a result of high crest velocities in the wave interacting with the resistance wires or excitation of the gantry and support structure for the wave gauges via wave loading on the fixed device. 

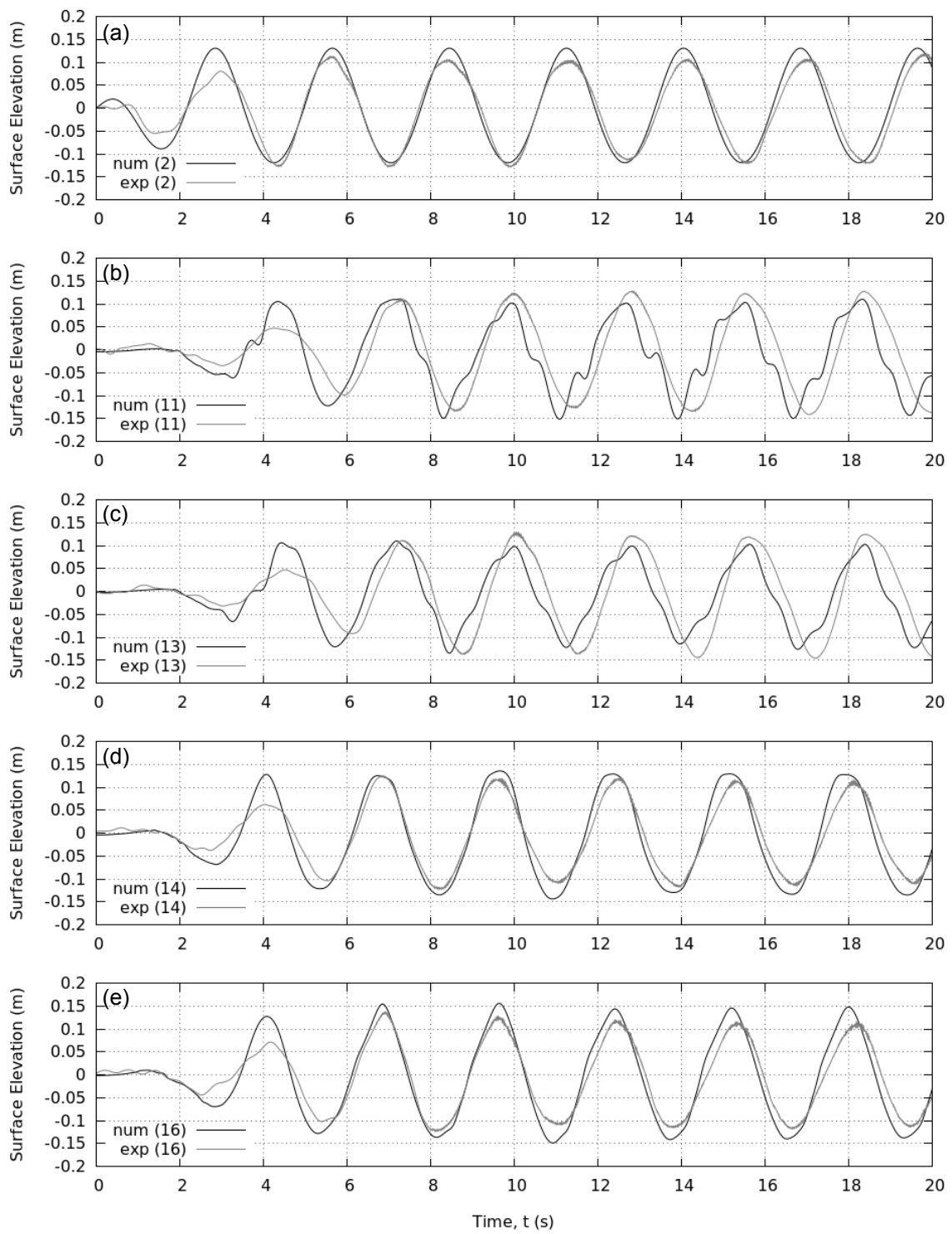

Figure 5: Surface elevation measurements for regular wave A and a fixed Wavestar device. Physical (grey) and numerical (black) results at locations (a) upstream of the float centre (2), (b-c) downstream of the float centre (11) and (13) and (d-e) along-crest of the float centre (14) and (16). See Figure 2 f for wave gauge locations. 

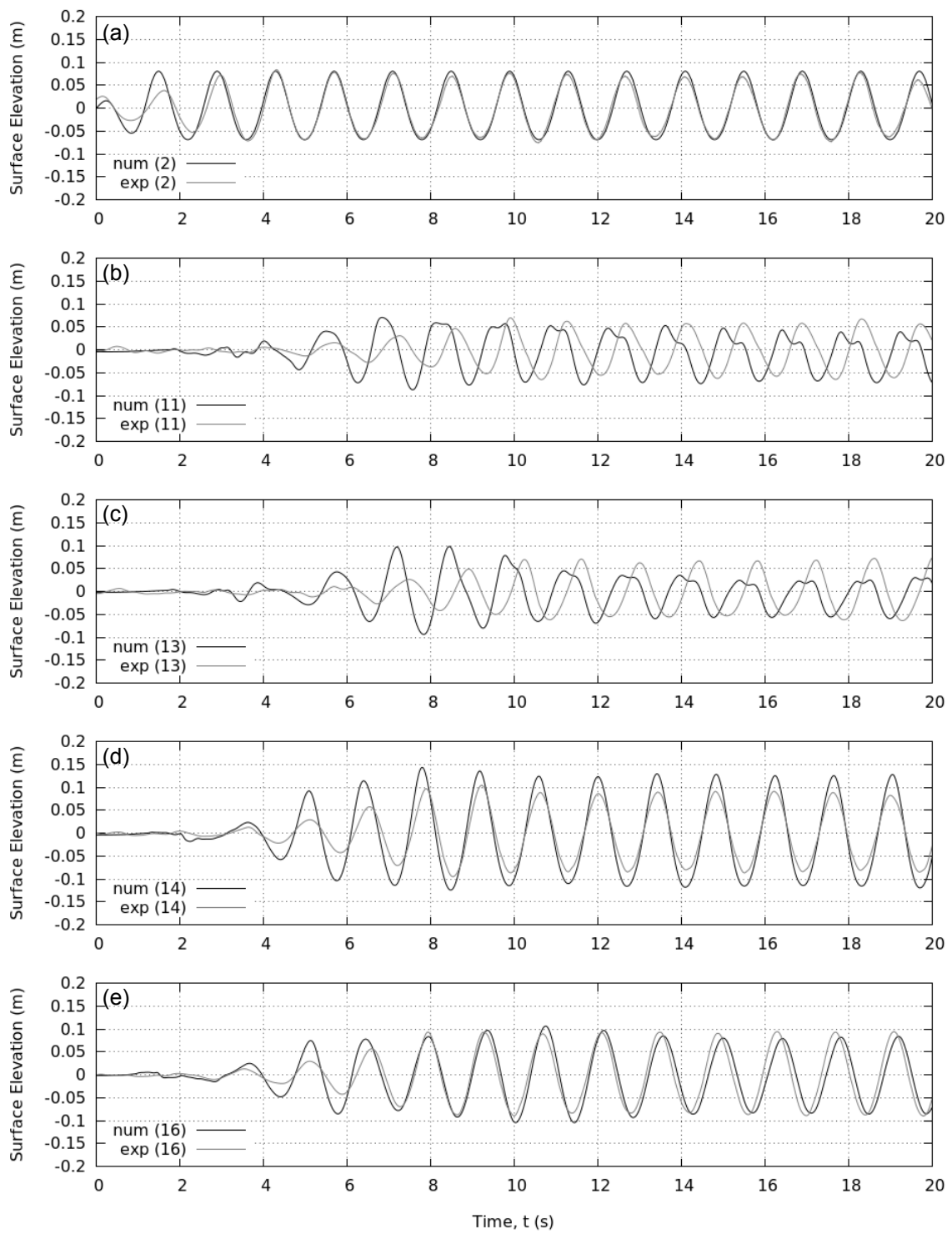

Figure 6: Surface elevation measurements for regular wave B and a fixed Wavestar device. Physical (grey) and numerical (black) results at locations (a) upstream of the float centre (2), (b-c) downstream of the float centre (11) and (13) and (d-e) along-crest of the float centre (14) and (16). See Figure 2 f for wave gauge locations. 
Plots (b) and (c), in Figures 5 and 6 , show the surface elevation in the wake region of the device. From the physical results in the higher frequency wave case (B) (Figure 6) it can be seen that the presence of the device disturbs the free surface downstream considerably; the wave height in this case is greatly reduced immediately behind the float (Figure $6 \mathrm{~b}$ and $6 \mathrm{p}$ ), suggesting significant diffraction effects. In the lower frequency wave case (A) (Figure 5 there is comparatively less disturbance in the physical surface elevation measurements behind the float (Figure $5 \mathrm{~b}$ and $5 \mathrm{p}$ ). One might expect this, as the wavelength of the higher frequency wave $(\mathrm{B})\left(\lambda_{B}=3.06 \mathrm{~m}\right)$ is much closer to the diameter of the float $(1 \mathrm{~m})$ than that of lower frequency wave $(\mathrm{A})\left(\lambda_{A}=11.39 \mathrm{~m}\right)$. However, the numerical results in the wake region are quantitatively quite poor. Although the reduction in wave height behind the device has been captured in case B (Figure 6), in both cases the numerical simulation predicts some higher frequency disturbances in the surface elevation and the phase of the resulting wave is incorrect. It would appear that the diffraction and scattering of waves has not been captured very well. However, it was observed that the physical device and support frame was not perfectly stiff and was able to move slightly, particularly in larger waves, producing radiated waves which may have affected these records. Also, at these locations, reflections from the tank walls $(7.75 \mathrm{~m}$ from the float centre) may have corrupted the results and some transverse waves were observed both up- and down-stream of the device after the larger wave experiments. As mentioned earlier, turbulence may play an important role in the wake region behind structures and so an investigation into appropriate turbulence modelling is required. It is also possible that an increased mesh resolution is needed around the structure, to capture higher-order free surface behaviour, as the one used here has been designed based on mesh independence in the wave-only case [14.

The final two plots ((d) and (e)), in Figures 5 and 6 , show the surface 325 elevation to the side (along-crest) of the float centre. At these locations one would expect to see the presence of any scattered (or radiated) waves from the float manifested as differences in the surface elevation compared to the incident 
wave (plot (a) in Figures 5 and 6). The physical results in Figures 5 and 6 suggest there is minimal scattering or radiating of waves from the structure (the time series' in plots (d) and (e) almost match the undisturbed incident time series in plot (a)). The only noticeable difference is a slight increase in the wave height to the side of the device in the higher frequency wave case (B) (plots 6d and 6e have greater amplitude than 6a). The numerical results, however, show significantly more evidence of scattering; the longer wave case 335 (A) shows flattening of the crests and troughs close to the float (Figure 5d) and sharpening of the crests and troughs further from the float (Figure 5); the shorter wave shows a much more exaggerated increase in wave height close to the float (Figure 6 d) and a slight drift out of phase further from the float (Figure 6e). It is likely that any scattered waves would be re-reflected from the sides 340 of the numerical domain (as there is no absorption there) and, due to concerns over CPU effort, the narrow domain may mean these reflected waves cause the observed discrepancies in the other time series.

In order to further investigate the discrepancies seen in the wake region, Figure 7 shows a spectral analysis of the free surface measurements taken directly behind the float at position 11 (plot b in Figures 5 and 6). Due to the relatively short length of time simulated in the numerical cases $(20 \mathrm{~s})$ the frequency resolution is fairly coarse. However, the numerical solution has accurately predicted the frequency of the main component in each case. The numerical solution has also predicted contributions at the higher harmonics of the main frequency in each case (up to the fourth harmonic). Most of these higher harmonic contributions are also present in the experimental data but have significantly lower amplitudes compared to those predicted by the numerical simulation. Curiously the third harmonic in the high frequency case (B) (Figure 7p) is present in the numerical solution but missing from the physical data. As mentioned above, 355 it is possible that a greater mesh resolution is required to correctly simulate the higher harmonics in the diffraction field behind the float. However, the observed over-estimation in amplitude at these frequencies might be expected if the physical model was able to move slightly during the experiment. 

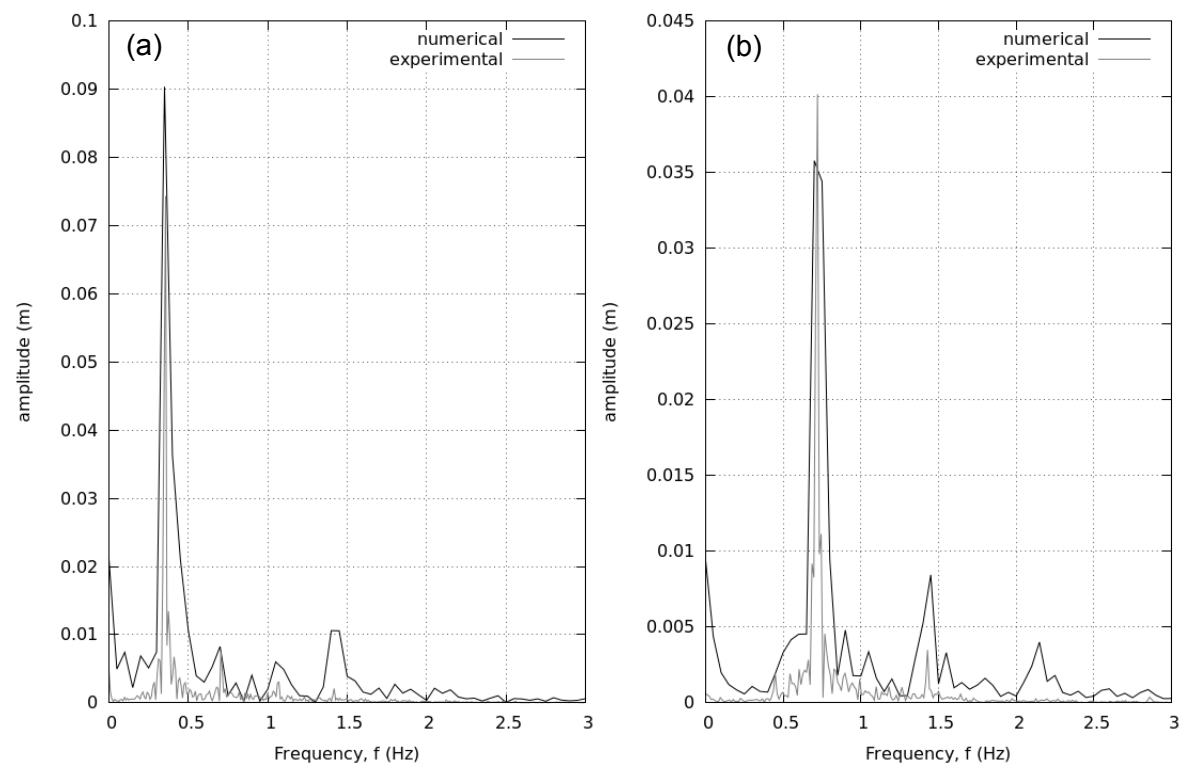

Figure 7: Frequency domain analysis of the surface elevation time series' measured directly downstream of the fixed Wavestar device at position 11, when subject to incident waves; (a) wave $\mathrm{A}$ and (b) wave $\mathrm{B}$.

Figures 8 and 9 show samples of the pressure results on the surface of the float compared with those recorded in the two physical experiments. The line numbers correspond to the probe labels in Figure $2 \mathrm{p}$, i.e. running from (a)(g): 22, 14 and 6 are on the up-stream side of the float at progressively greater submergence below the still water level, 1 is at the bottom centre of the float and 2, 10 and 18 are on the downstream side of the float with progressively lower submergence.

By considering the longer wave (A) results in Figure 8 first, we can see that the numerical solution is reasonably good. Bearing in mind that the physical results plotted here are raw calibrated measurements, there are very few differences. Generally the numerical solution has a tendency to over-estimate the depth of the troughs but the peaks show very good agreement (except in Figure 8a). An explanation for the discrepancies in the Figure 8 a could be that 

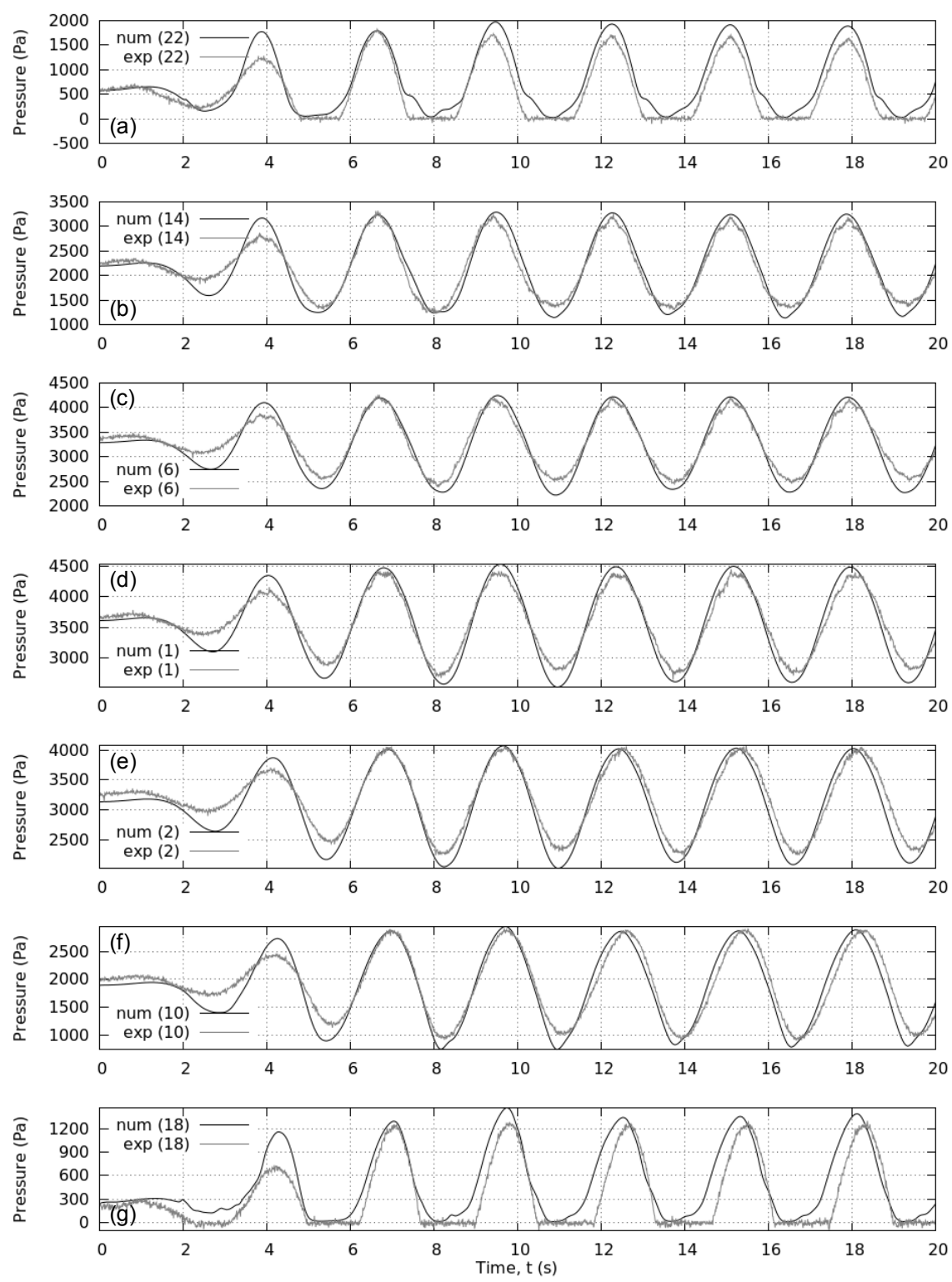

Figure 8: Pressure measurements for regular wave A and a fixed Wavestar device. Physical (grey) and numerical (black) results at positions on the surface of the float along a line running from front to back parallel to the direction of wave propagation. See Figure $2 \mathrm{~b}$ for probe locations. 

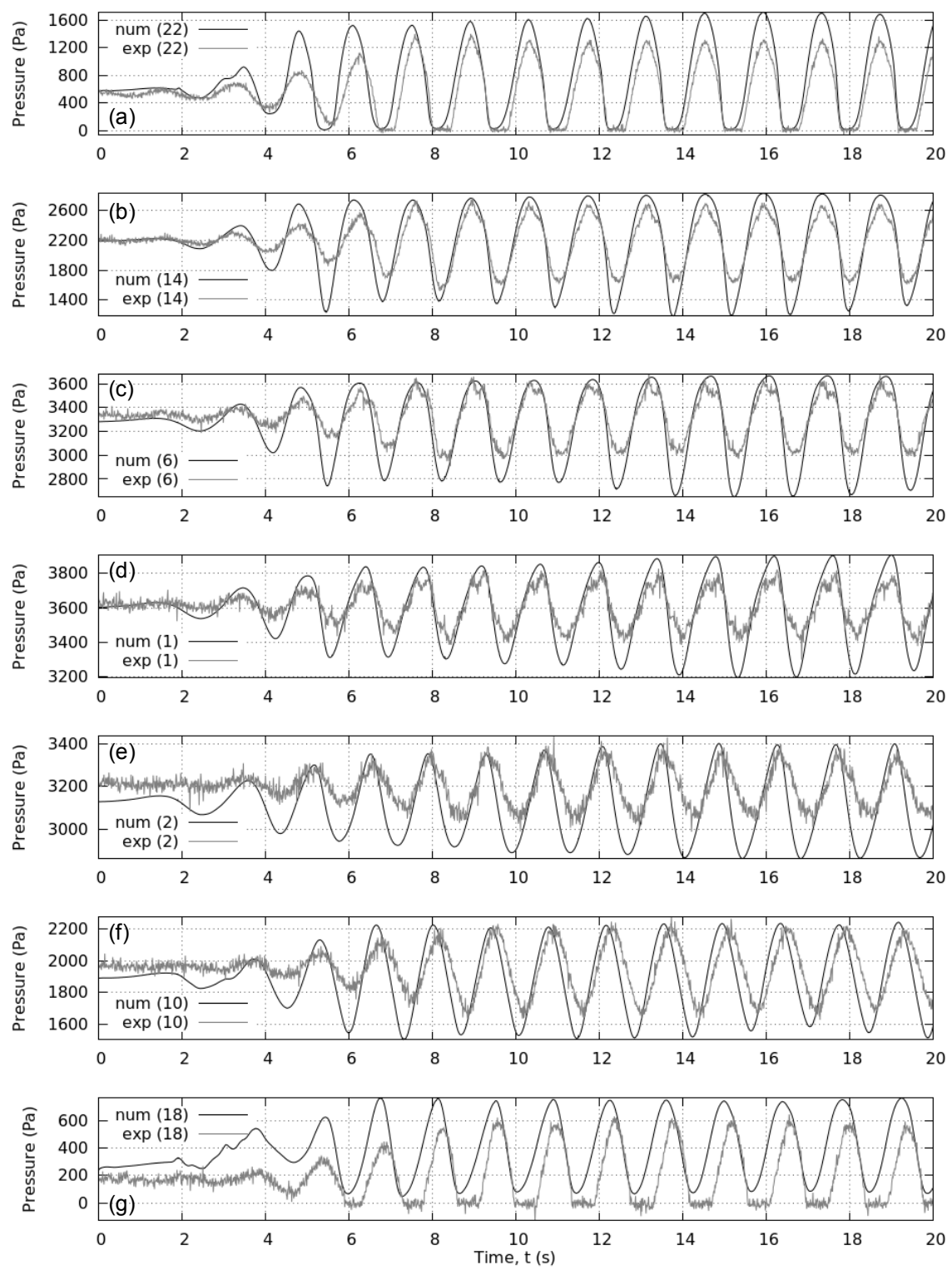

Figure 9: Pressure measurements for regular wave B and a fixed Wavestar device. Physical (grey) and numerical (black) results at positions on the surface of the float along a line running from front to back parallel to the direction of wave propagation. See Figure $2 \mathrm{~b}$ for probe locations. 
this position is very close to the free surface (evident by the flat troughs at $0 \mathrm{~Pa}$ showing the probe dried at these times) and so any complex behaviour in the interface region may have been lost due to the $\mathrm{VOF}$ treatment of the free surface. Furthermore, the surface elevation results hinted at the numerical simulation having a slightly greater incident wave height which may explain any over-estimations in the pressure. Despite this, the probe at position 18 (Figure $8 \mathrm{~g}$ ) shows the greatest discrepancy between the physical and numerical results. Although 18 is also near the free-surface there appears to be an additional phase shift relative to the experimental data as well as some high frequency disturbances. This position is on the downstream side of the float, in the wake region, and so this discrepancy is in keeping with the phase shift and over-predicted higher harmonics observed in the surface elevation results. For the shorter wave (B), in Figure 9, the comparison between the numerical and physical results is 385 far worse, however, the physical pressure measurements are very 'noisy' and so there is some doubt over their precise amplitude. The numerical simulation appears to capture the main behaviour but tends to overestimate the amplitude of the pressure fluctuations. Again, a phase shift relative to the experimental data is observed on the downstream side of the float. It is clear that, in this work, the numerical model has some difficulties reproducing the flow in the wake of the float.

Figures 10 and 11 show the velocity measurements, for the two wave cases, at the ADV position in the along-crest direction from the float centre (between wave gauges 15 and 16 in Figure 22). In both cases the numerical solution predicts the same behaviour as in the experiments. In general, however, the amplitude of the vertical velocity fluctuation (Figures 10 and 11 a) is overestimated numerically whilst the horizontal velocity in the direction of incident wave propagation, $V_{x}$, is underestimated in the longer wave case (Figure 10b) but a good match in the shorter wave case (Figure 11b). Of most interest, however, is the velocity perpendicular to the incident wave propagation, $V_{y}$, which, although much smaller in amplitude, displays a crucial difference between the numerical and physical results in the longer wave case (Figure 10;). In unidi- 

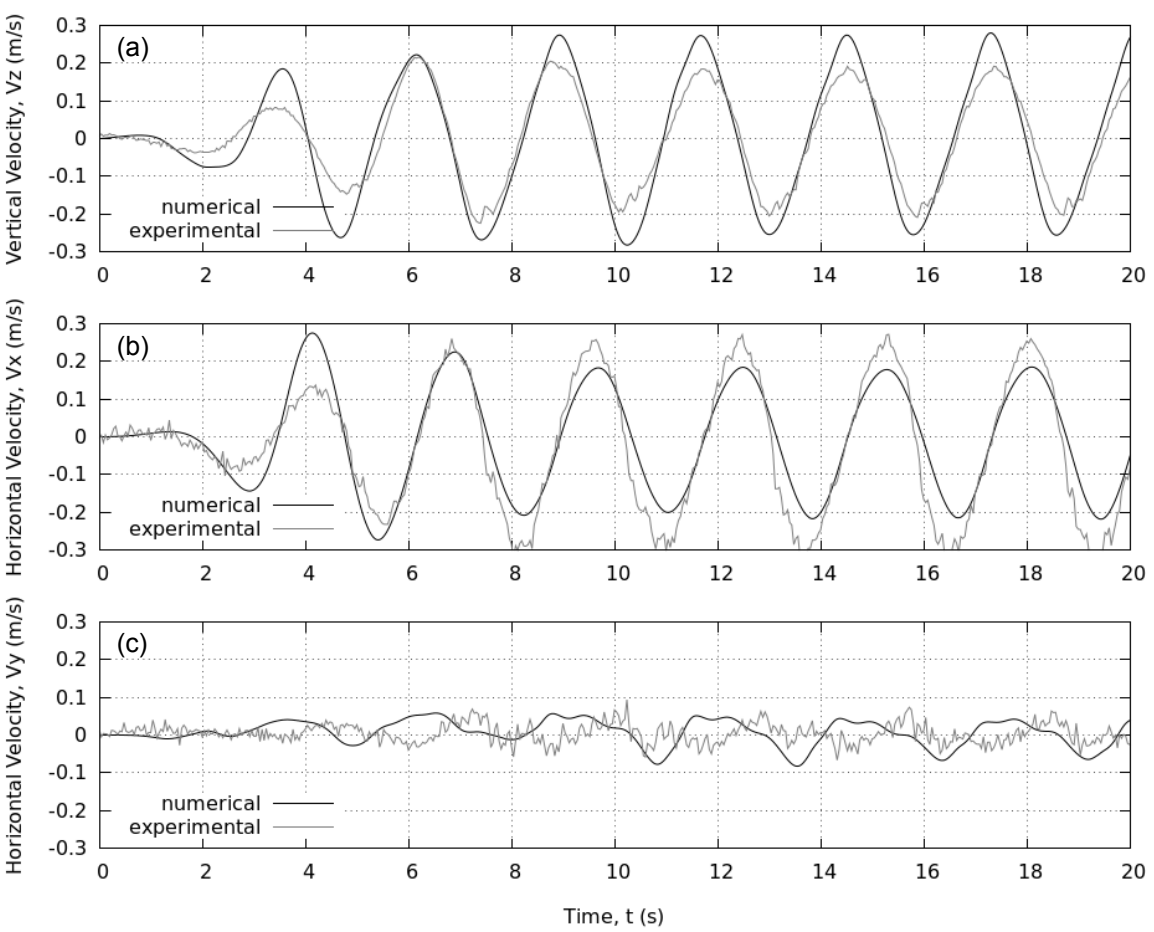

Figure 10: Velocity measurements for regular wave A and a fixed Wavestar device. Physical (grey) and numerical (black) results at the along-crest Vectrino position (Figure 2f). (a) Vertical velocity, $\mathrm{Vz}$; (b) horizontal velocity in direction of incident wave propagation, Vx; (c) horizontal velocity perpendicular to direction of incident wave propagation, Vy.

rectional waves $V_{y}$ should be zero, therefore any variation in $V_{y}$ must be as a direct result of waves scattered (or radiated) from the device and this is likely to be heavily influenced by the over-predicted higher harmonics observed earlier (Figure 7). Despite this, in the shorter wave case (Figure 11k) the numerical solution predicts $V_{y}$ and the presence of the scattered wave well, with a slight overestimation of the amplitude; in the longer wave case (Figure 10;), however, the amplitude of $V_{y}$ is correct but the scattered wave appears to arrive out of phase compared with the physical measurements. It is possible that the differences in the scattered wave could go some way to explain the difference in surface elevation and vertical velocity in the along-crest direction from the float. 

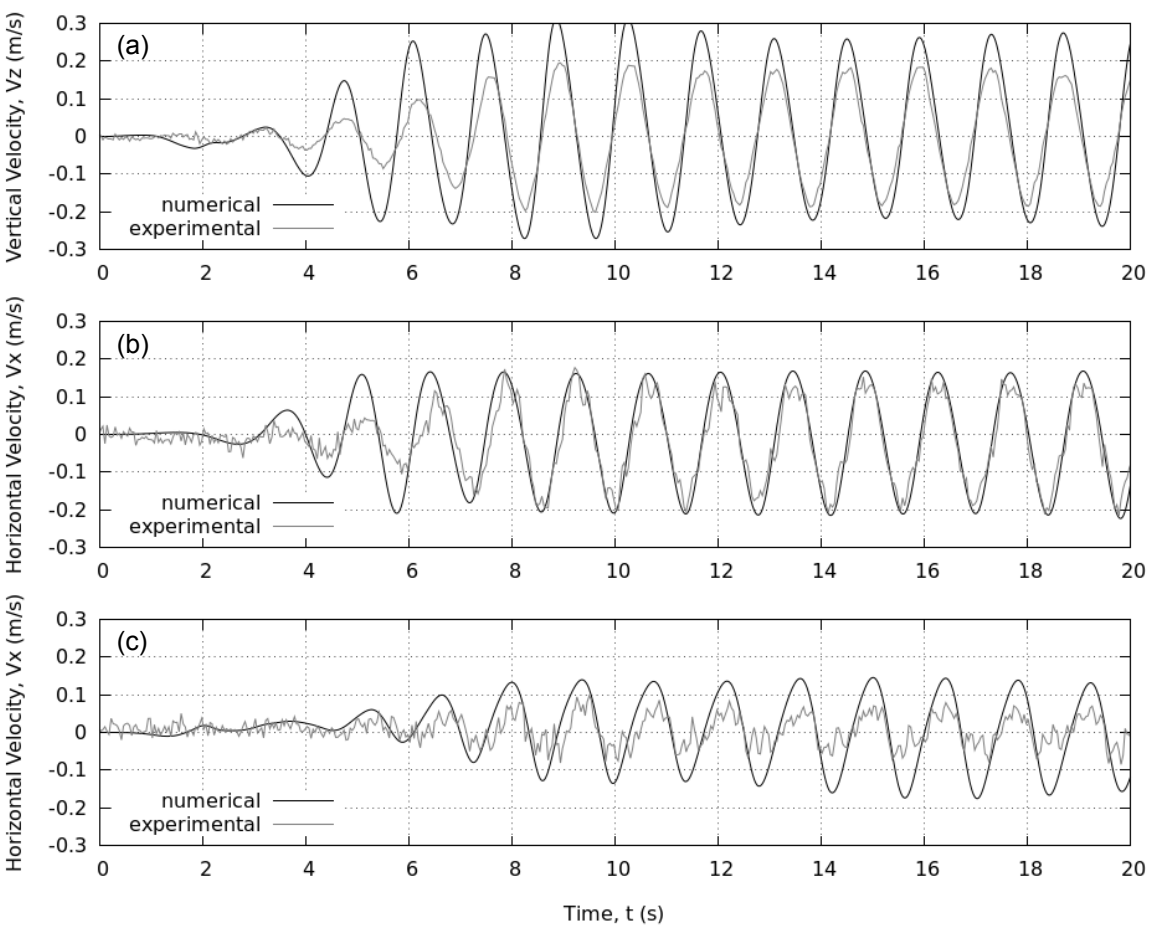

Figure 11: Velocity measurements for regular wave B and a fixed Wavestar device. Physical (grey) and numerical (black) results at the along-crest Vectrino position (Figure 25). (a) Vertical velocity, $\mathrm{Vz}$; (b) horizontal velocity in direction of incident wave propagation, Vx; (c) horizontal velocity perpendicular to direction of incident wave propagation, Vy.

For example, in the shorter wave case (Figure 11k) the numerical solution for $V_{y}$ predicts a higher amplitude scattered wave than in the physical experiment and, arriving in phase at the ADV position, this will constructively interfere with the incident wave to produce an overestimation of vertical velocity and surface elevation at this location. Furthermore, the differences in the scattered wave observed in the long wave case (Figure 10 c) could explain the unexpected flattening and sharpening of the surface elevation results to the side of the float (Figure 5d-e). An additional concern is that, because there is no absorption on the side walls of the NWT, any scattered waves will be re-reflected and are likely to affect the results in the test area. This is also the case in the physical 

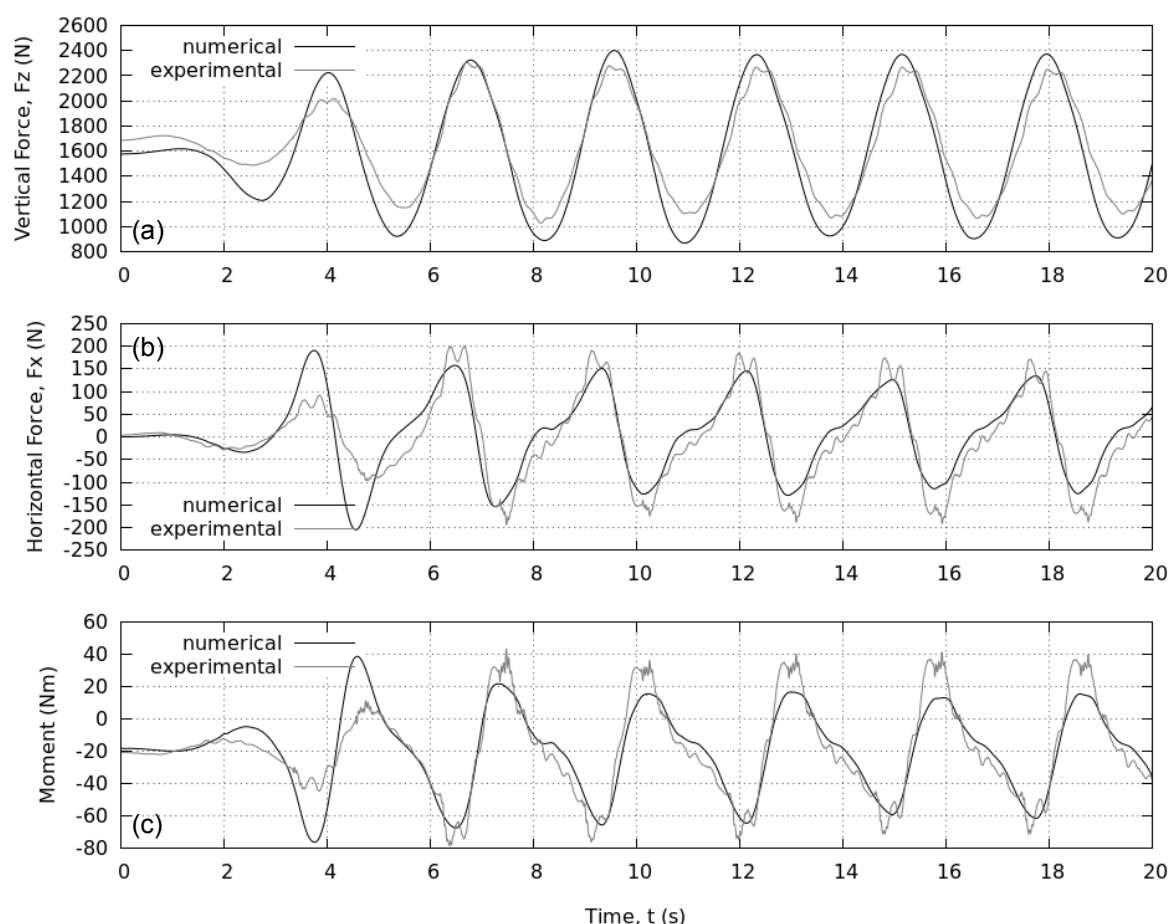

Figure 12: Force and moment on a fixed Wavestar device in regular wave A. Physical (grey) and numerical (black) results measured at the $6 \mathrm{DOF}$ force and torque just above the float (Figure 2a). (a) Vertical force, Fz; (b) horizontal force in direction of incident wave propagation, Fx; (c) moment about sensor location.

experiments, however, in the numerical case the side walls are much closer to the test area and so this effect is not only different but compounded in the numerical simulation.

Lastly, Figures 12 and 13 show the force and moment measurements for the two wave cases at the $6 \mathrm{DOF}$ force and torque sensor positioned just above the float (Figure 2a). In each figure, Plot (a) shows a time series of the vertical force on the float; Plot (b) shows the horizontal force in the direction of wave propagation; and Plot (c) shows the moment about a horizontal axis perpendicular to the incident wave direction. In each case the experimental data has been transformed to the global coordinate system based on the orientation derived 
from the pressure measurements in still water. The zero offset has been applied based on the buoyancy force expected from Archimedes principle as a true zero offset measurement was not possible during the physical experiments.

Despite the over-predicted higher harmonics in the surface elevation behind the float (Figure 7), for the longer wave case considered (A) (Figure 12 the agreement between the numerical and experimental results is very good (after the first wave cycle). The amplitude of the vertical force is over-estimated but this is again in keeping for the slightly greater incident wave height observed in Figure 5 . The agreement for both horizontal force and moment is also very good, particularly taking into account the unfiltered nature of the physical results. One further observation is that the numerical solution has slightly underestimated the expected buoyancy force in still water (offset in vertical force ${ }_{445}$ at $t=0 \mathrm{~s}$ ). It is possible that, as discussed when considering the pressure results, surface-piercing structures suffer from inaccuracies caused by the finite width of the interface region and this may have affected the buoyancy force.

For the shorter wave case (B) shown in Figure 13 the agreement is not so good. As with the velocities and pressures, the numerical simulation tends to over-estimate the amplitude of the forces and moment. The correct frequency is observed and the slight asymmetry of the crests in both the horizontal force and moment time series is also reproduced, i.e. the horizontal force decreases more rapidly than it increases while the moment increases more rapidly than it decreases. In both the pressure and velocity measurements for this case, the experimental data has high frequency distortion throughout the time series. This could reduce confidence in the experimental results or be evidence of turbulence resulting from the wave-body interaction. Reviewing video footage of the experiments, the water was not completely still at the beginning of each experiment which may have contributed to the discrepancies observed in these comparisons, particularly in the smaller shorter wave case.

Despite the discrepancies discussed above, the NWT has performed very well, predicting the pressure and load on the float, as well as the surface elevation and velocity in the vicinity of the structure, to a good degree of accuracy. It is 

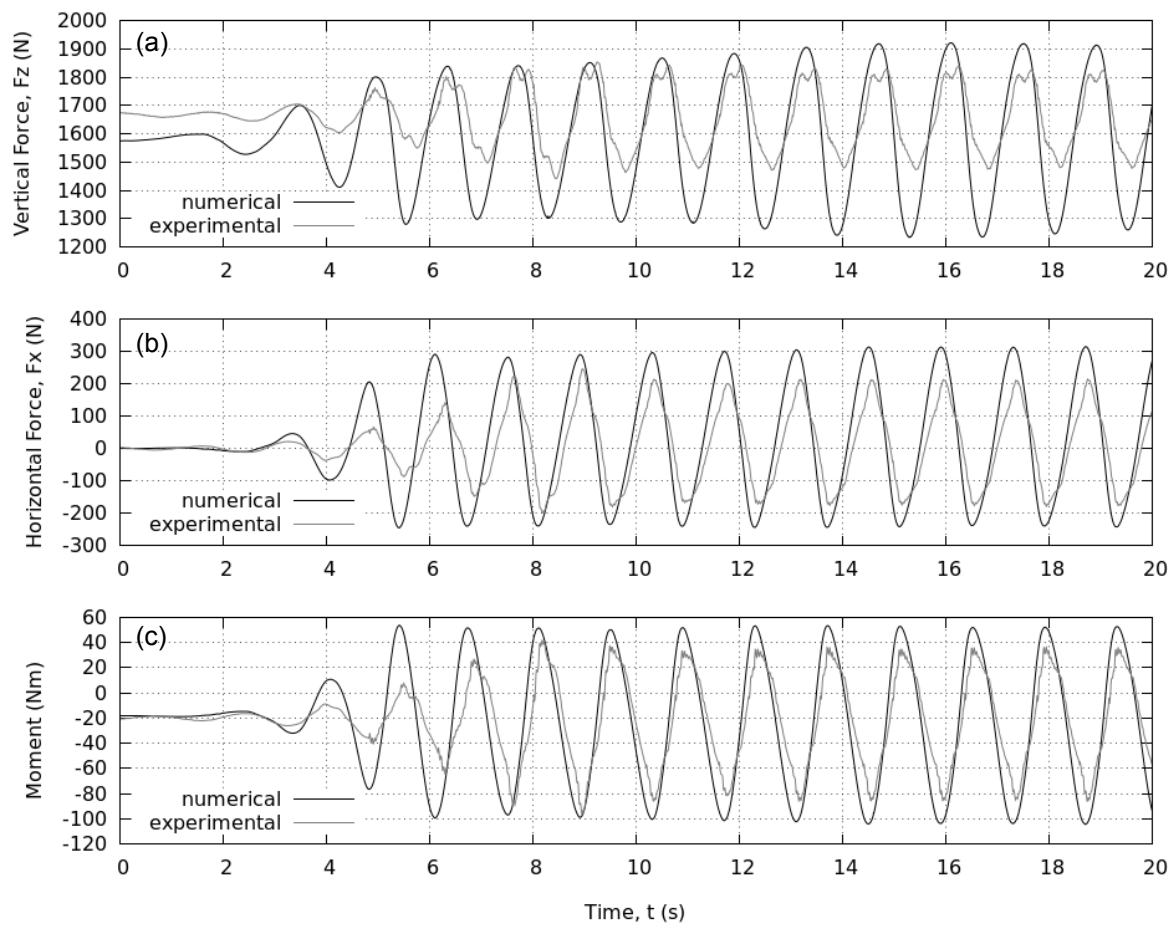

Figure 13: Force and moment on a fixed Wavestar device in regular wave B. Physical (grey) and numerical (black) results measured at the $6 \mathrm{DOF}$ force and torque just above the float (Figure 2a). (a) Vertical force, Fz; (b) horizontal force in direction of incident wave propagation, Fx; (c) moment about sensor location.

possible that the performance is slightly decreased for higher frequency incident waves and there is still some concern over the quality of the numerical result in the wake region behind the float. However, for fixed structures, the NWT has been proven to produce reliable results for some relatively complex flow phenomena.

Lastly, the execution times for the two 20 s simulations were 23.75 and 26.77 hours for wave A and B respectively, each running on 2 Intel ${ }^{\circledR}$ Xeon(R) CPU E5630 @ $2.53 \mathrm{GHz}$ processors. This may suggest higher frequency waves require greater CPU effort, but, an execution time of 1 day, using minimum resources, is considered to be acceptable for such a complex CFD simulation. 


\subsection{Freely pitching cases}

Allowing for coupled motion, the same two wave cases have been simulated with the device able to move freely about the hinge point with the $\mathrm{PTO}$ system disabled. The computational domain and the device's starting position were identical to those used in the fixed cases.

Due to the constraints on the device's motion, its position can be transformed

480 Figure 14 shows both the experimental and numerical displacement in the cylinder during the longer wave case (A). Here a positive displacement corresponds to a lifting of the device, i.e. a crest. Clearly, according to the numerical results, the device was not initially set at its neutrally-buoyant position. This

However the numerical simulation over-estimates the amplitude of the oscillation and also predicts a slight asymmetry in the motion which is not observed in the physical results. It is known from the experiments, however, that the motion of the physical device was slightly damped by frictional forces in the 495 frequency of the motion in the physical case. A possible alternative explanation could be that due to the increased proximity of the side walls in the NWT any scattered or radiated waves coming from the device will return to the test area with greater amplitude and interfere differently with the incident wave. Conlead to increased motion of the device. There does appear to be an unexpected increase in the amplitude of motion after the first stable cycles which may be evidence of the arrival of reflected waves once the device has started to oscillate. It is likely that this effect is dependent on the relationship between the radiated 


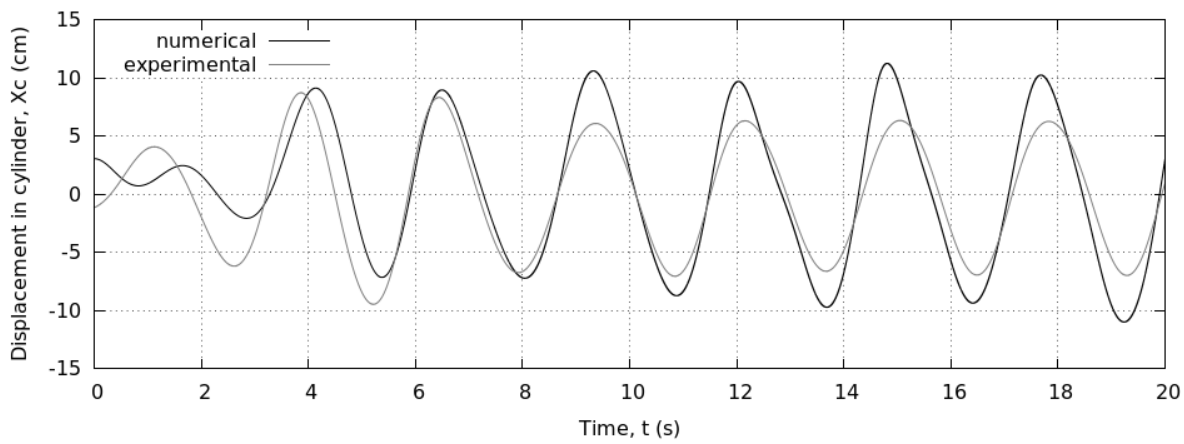

Figure 14: Comparison between experimental (grey) and numerical (black) results for the displacement in the cylinder of the Wavestar device with the PTO disabled and when subject to regular waves $\mathrm{A}$. and Column $3(i=3)$ shows the difference (numerical - physical) between the 

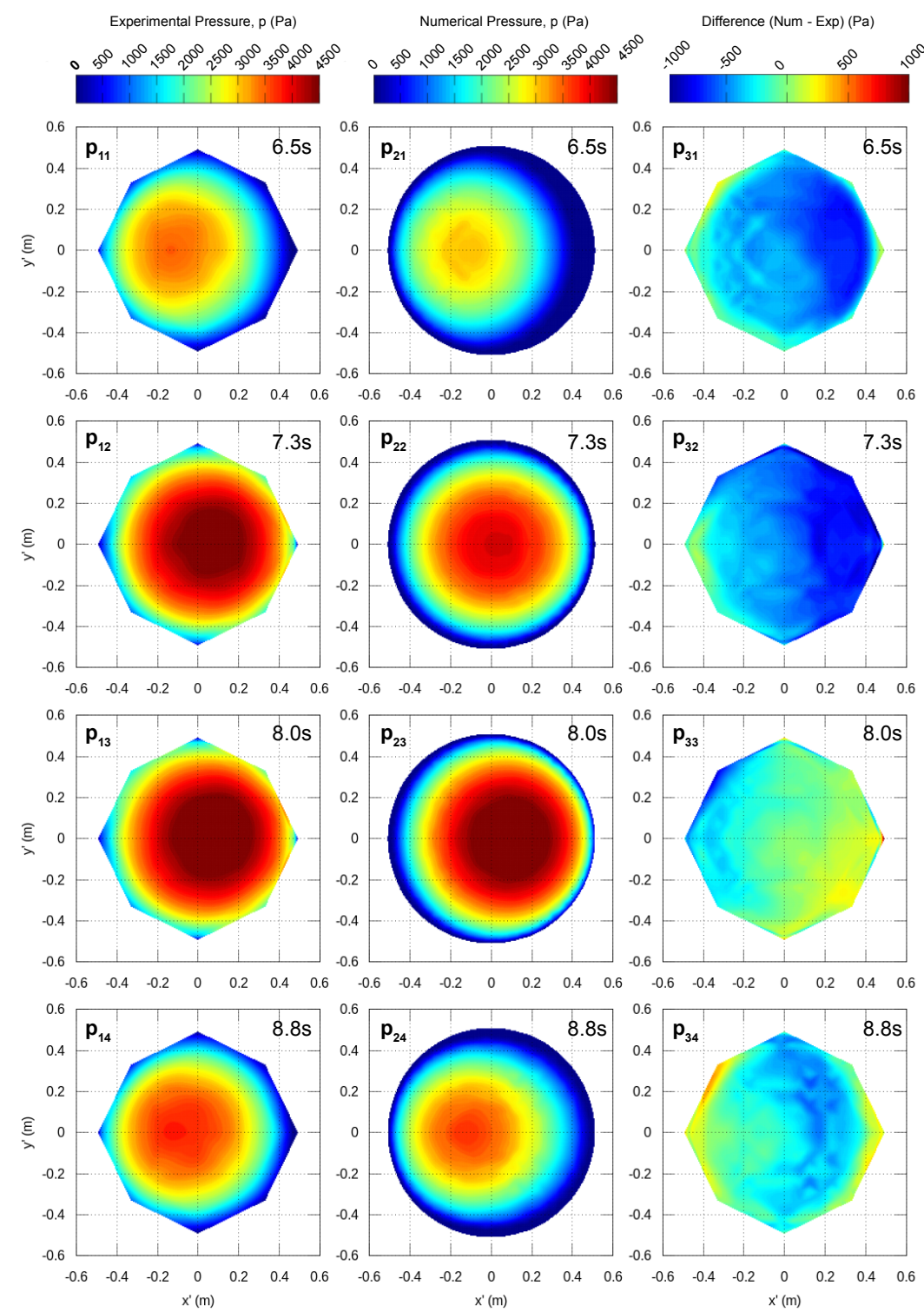

Figure 15: Distribution of pressure, projected onto a 2D Cartesian plane, in the frame of reference of the Wavestar float, during regular wave A. Experimental (column 1), numerical (column 2) and difference (column 3) at discrete times at a crest (row 1), falling (row 2), in a trough (row 3) and rising (row 4). 
two. Row $1(j=1)$ is at a time close to a peak in the displacement, Row 2 $(j=2)$ is at a time on the falling side of a peak, Row $3(j=3)$ is at a time close to a trough in the displacement and Row $4(j=4)$ is at a time on the rising side of a peak. In general, the numerical result is good. It appears that there is an overestimation during a trough $\left(p_{33}\right)$ and an underestimation during a peak $\left(p_{31}\right)$. This is in keeping with the overestimated amplitude of motion response observed in Figure 14 and a number of discrepancies might be explained by the difference in motion. It might be speculated that the greatest differences occur on the positive $x^{\prime}$ side (the back) of the float which supports the observed inaccuracies in the wake region discussed in the fixed cases.

For the steeper wave case (B), the results for the displacement, $X_{c}$, are shown in Figure 16. Again, the numerical device does not begin in its neutrally buoyant position but after some time the motion is forced at the correct frequency by the incident wave. In this case the experimental results display some low frequency beating of the $X_{c}$ displacement which can only be explained by reflected waves in the physical basin. The numerical result shows some similar behaviour but begins by over-estimating the amplitude of the motion and then under-estimates it after a period of comparable motion. If this behaviour is a result of reflections it is not surprising that there are differences between the numerical case and the physical case which has a much greater domain size. However, it is expected that the radiated, and so reflected, waves would have the same frequency as the incident wave and so the phase relationship, and hence the interference, should result in a constant modulation of the incident wave at the float position (assuming the float behaviour is a function of events at a point at its centre). The beating behaviour is typically associated with a combination of slightly differing frequencies, the origin of which has not been identified in this case. Again, no further time instances have been simulated.

The equivalent pressure results for this steeper wave are shown in Figure 17. Again the numerical result is good. In this case the pressure is consistently under-estimated but it is likely that this can be explained by the difference in motion/position of the device (Figure 16) at the specific times plotted. Again, 


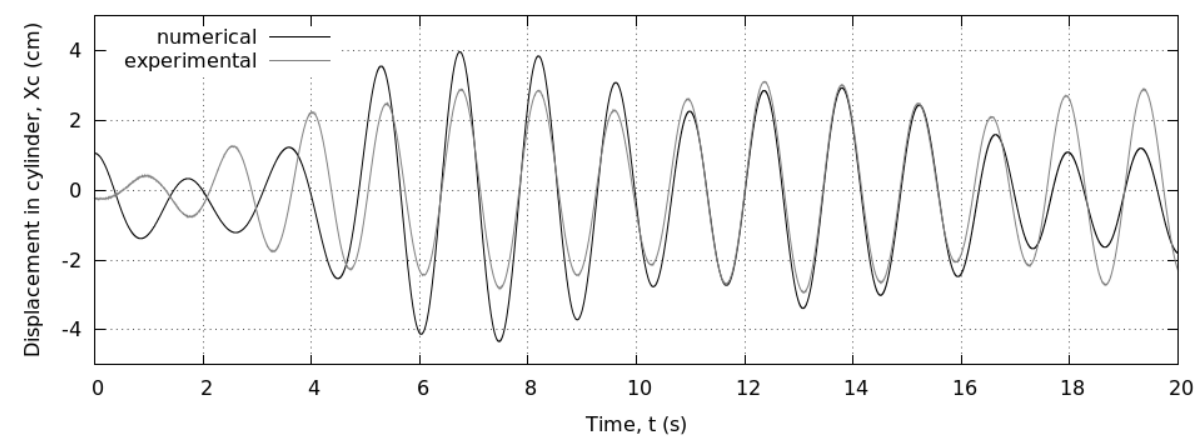

Figure 16: Comparison between experimental (grey) and numerical (black) results for the displacement in the cylinder of the Wavestar device with the PTO disabled and when subject to regular waves $\mathrm{B}$

it seems as though the greatest discrepancies are present on the back of the float further highlighting that additional work is required to correctly simulate the fluid behaviour in the wake of the device.

Lastly, the execution times for the two 20 s simulations were 137.5 and 56.34 hours for wave A and B respectively, each running on 6 Intel ${ }^{\circledR}$ Xeon(R) CPU E5630 @ $2.53 \mathrm{GHz}$ processors. This may suggest that the amplitude of oscillation, and hence the mesh deformation, has a bigger effect on the CPU effort than the wave frequency does. Also, with the addition of mesh motion and the associated hydrodynamics, the execution time has increased dramatically compared to the fixed cases. For the larger wave case (A), the solution for a moving structure takes almost six times longer than that with the structure fixed, even with triple the processing power.

\subsection{Further numerical investigation - extreme waves}

In order to test the robustness of the $\mathrm{NWT}$ and $6 \mathrm{DOF}$ solver, a very steep regular wave was simulated with the device still able to move freely. The wave had a $2 \mathrm{~s}$ period, a height of $0.68 \mathrm{~m}$ and a steepness $(H / \lambda)$ of 0.11 . It was also part of the tests performed by 33 . However, due to fears over possible damage to the device due to excessive motion, physical tests with this wave were not 

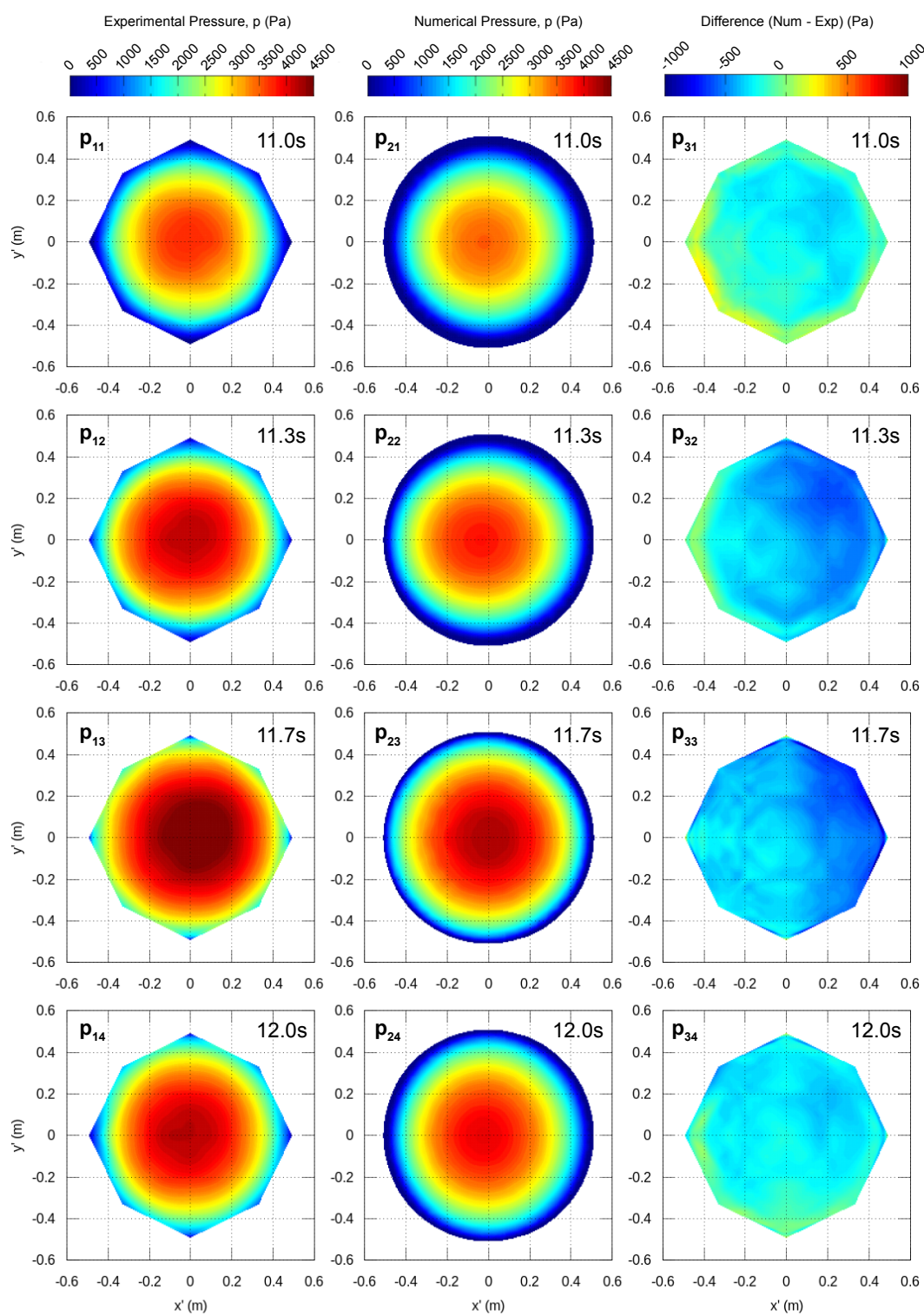

Figure 17: Distribution of pressure, projected onto a 2D Cartesian plane, in the frame of reference of the Wavestar float, during regular wave B. Experimental (column 1), numerical (column 2) and difference (column 3) at discrete times at a crest (row 1), falling (row 2), in a trough (row 3) and rising (row 4). 


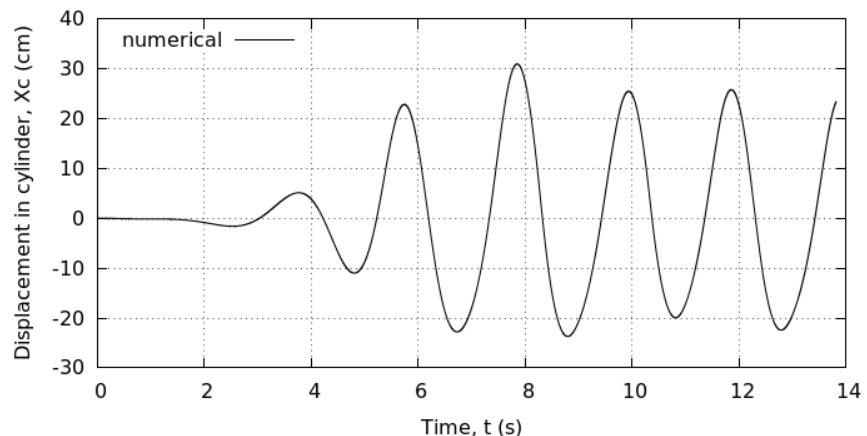

Figure 18: Numerical results for the displacement in the cylinder of the Wavestar device with the PTO disabled and when subject to extreme regular waves

performed with the PTO system disabled. Therefore, there is no physical data for the freely oscillating device in this case. Despite this, a numerical simulation was performed using the same NWT design as for the other free cases and the resulting displacement in the cylinder, $X_{c}$, is shown in Figure18, As can be seen, the motion of the device is considerable (more than double the displacement seen in case A). In addition to this, Figure 19 shows a series of snapshots from the simulation including the maximum elevation (b), the minimum elevation (d), and positions between these ((a) and (c)). It can be seen that, during a wave cycle the float goes from being completely submerged to nearly leaving the water altogether. The fluid behaviour is correspondingly complex with high levels of free surface distortion, radiated waves, green water, spray and recombination. It is encouraging that the NWT has handled all of these without issue and that the end result appears to be qualitatively reasonable but physical validation is still required for cases with such extreme motion.

Lastly, the execution times for the $13.82 \mathrm{~s}$ simulation was 185 hours, running on 6 Intel ${ }^{\circledR}$ Xeon(R) CPU E5630 @ $2.53 \mathrm{GHz}$ processors. The large wave case above (A) took only 73 hours to reach $13.82 \mathrm{~s}$ using the same hardware. This is further evidence, that the amplitude of oscillation and the associated complex hydrodynamics is critical in the CPU effort required to complete the simulation. 

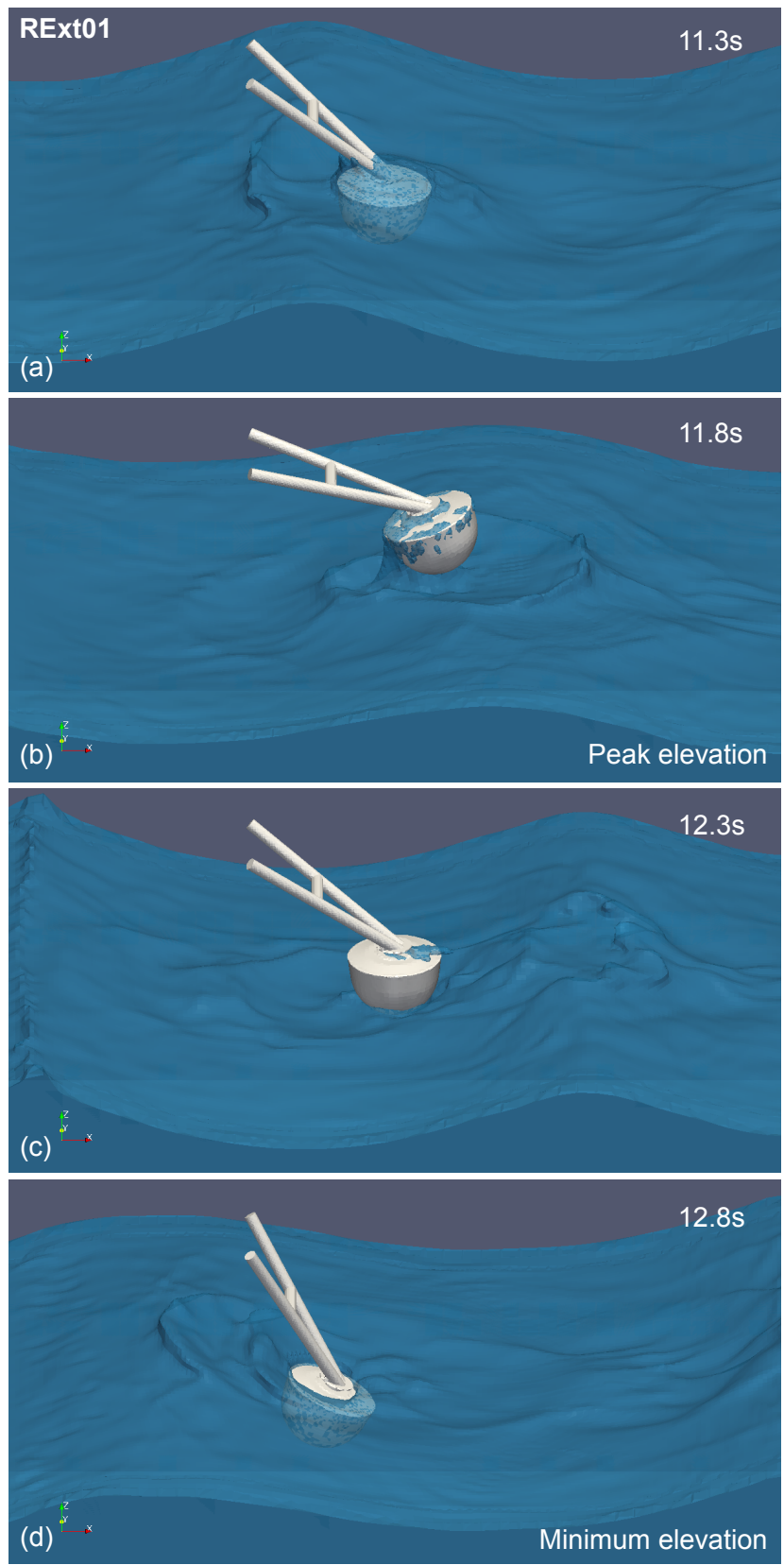

Figure 19: Snapshots of a freely moving Wavestar in extreme regular waves (RExt01). 


\section{Conclusions}

In conclusion, with a significant increase in the complexity of the geometry over similar cases in the literature, OpenFOAM ${ }^{\circledR}$ and the ${ }^{\mathrm{NWT}}$ developed by Ransley 14 performs well for cases with a fixed structure. The pressure distribution and loading on the Wavestar device is reproduced accurately. There appear to be some issues in the wake region behind the device; high frequency disturbances are present in the surface elevation behind the float and both the pressure and surface elevation results in this region exhibit a noticeable phase shift compared with the experiment. Furthermore, based on velocity measurements to the side of the float, the scattered waves are not reproduced correctly. A greater mesh resolution may be required to capture higher-order free surface behaviour in the vicinity of the structure, however, the observed discrepancies may also be a result of reflected waves in the domain. The quality of the results does not appear to reduce with an increase in wave steepness although only two cases have been studied here.

For freely pitching cases, the comparison between the physical and numerical results is also promising. There are differences in the pressure distribution and the motion of the device but these can generally be attributed to issues with reflected waves and the assumption that the motion in the physical experiment is completely undamped with the PTO disabled. Some unexplained 'beating' in the motion is observed for the higher frequency case but in general the behaviour of the device is captured well. As with the fixed case, the quality of the results does not appear to reduce with an increase in wave steepness. However, the execution time of simulations appears to increase significantly with greater device motion.

Finally, the NWT has been shown to remain stable through extreme motions including full submersion of the float, green water, break-up and recombination as well as slamming motions and strong radiation. All of which are likely to be important in the assessment of a device's behaviour and survivability at sea. 
The authors would like to acknowledge the COAST Laboratory technical staff and colleagues at Aalborg University, Denmark, for their assistance in designing and running the physical experiments and the Marine Renewables Infrastructure Network (MARINET) for funding the tests. The numerical work has been funded by the Engineering and Physical Sciences Research Council (EPSRC) via the SuperGen UK Centre for Marine Energy Research (UKCMER) as part of the third phase of the consortium's program of work (October 2011 September 2016).

\section{References}

[1] J. Cruz, Ocean Wave Energy. Current Status and Furture Perspectives, Springer, 2008.

[2] BMT Fluid Mechanics Ltd, Review of model testing requirements for FPSO's, Health and Safety Executive, 2001.

[3] The Carbon Trust, Guidelines on design and operation of wave energy converters, 2005.

[4] J. Morison, M. P. O'Brien, J. W. Johnson, S. A. Schaaf, The forces exerted by surface waves on piles, Journal of Petroleum Technology 2 (5) (1950) 149-154.

[5] WAMIT Inc., WAMIT Inc. The state of the art in wave interaction analysis, website, http://www . wamit.com/ accessed 7th January 2015, 2015.

q [6] ANSYS, Inc., ANSYS Aqwa, website, http://www.ansys.com/Products/ Other+Products/ANSYS+AQWA accessed 7th January 2015, 2015.

[ [7] Orcina Ltd., Orcina ... home of OrcaFlex, website, http://www.orcina. com/index.php accessed 7th January 2015, 2015. 
[8] K. Ruehl, D. Bull, Wave Energy Development Roadmap: Design to commercialization, in: Harnessing the Power of the Ocean, proceedings of OCEANS '12, Hampton Road, VA, 1-10, 2012.

[9] J. H. Ferziger, M. Perić, Computational Methods for Fluid Dynamics, Springer, 3 edn., 2002.

[10] M. Kramer, L. Marquis, P. Frigaard, Performance Evaluation of the Wavestar Prototype, in: Proceedings of the 9th European Wave and Tidal Conference (EWTEC), Southamption, UK, 2011.

[11] S. Ambühl, M. Kramer, J. P. Kofoed, J. D. Sørensen, Reliability Assessment of Wave Energy Devices, in: Proceedings of the 4th International Conference on Ocean Energy (ICOE), Dublin, Ireland, 2012.

[12] E. Vidal, R. H. Hansen, M. Kramer, Early Performance Assessment of the Electrical Output of Wavestars prototype, in: Proceedings of the 4th International Conference on Ocean Energy (ICOE), Dublin, Ireland, 2012.

[13] M. M. Jakobsen, S. Beatty, G. Iglesias, M. M. Kramer, Characterization of loads on a hemispherical point absorber wave energy converter, International Journal of Marine Energy 13 (2016) 1-15.

[14] E. Ransley, Survivability of Wave Energy Converter and Mooring Coupled System using CFD, Ph.D. thesis, Plymouth University, 2015.

[15] M. M. Jakobsen, Wave-Structure interactions on point absorber, Tech. Rep., Aalborg University, Denmark, 2014.

[16] L. Qian, D. M. Causon, C. G. Mingham, D. M. Ingram, A free-surface capturing method for two fluid flows with moving bodies, Proceedings of the Royal Society A (2006) 21-42.

[17] K. M. T. Kleefsman, G. Fekken, A. E. P. Veldman, B. Iwanowski, B. Buchner, A Volume-of-Fluid based simulation method for wave impact problems, Journal of Computational Physics 206 (2005) 363-393. 
[18] Y. Zhang, Q. Zou, D. M. Greaves, D. Reeve, A. Hunt-Raby, D. Graham, P. James, X. Lv, A Level Set Immersed Boundary Method for Water Entry and Exit, Communications in Computational Physics 8 (2) (2010) 265-288.

[19] J. Westphalen, D. Greaves, C. Williams, K. Drake, P. Taylor, Numerical simulation of an oscillating cone at the water surface using computational fluid dynamics, in: Proceedings of International Workshop on water waves and floating bodies, Zelegnorsk, Russia, 2009.

[20] J. Westphalen, Extreme Wave Loading on Offshore Wave Energy Devices using CFD, Ph.D. thesis, Plymouth University, 2010.

[21] E. P. Bangun, T. Utsunomiya, Evaluation of Viscous Forces Acting on A Moving Body by Navier-Stokes Solver, in: Proceedings of OCEANS 2008, Kobe, Japan, 1-8, 2008.

[22] E. B. Agamloh, A. K. Wallace, A. von Jouanne, Application of fluidstructure interaction simulation of an ocean wave energy extraction device, Renewable Energy 433 (2008) 748-757.

[23] Z. Z. Hu, D. M. Causon, C. G. Mingham, L. Qian, Numerical simulation of floating bodies in extreme free surface waves, Natural Hazards and Earth System Sciences 11 (2011) 519-527.

[24] M. A. Bhinder, C. G. Mingham, D. M. Causon, M. T. Rahmati, G. A. Aggidis, R. V. Chaplin, Numerical and Experimental Study of a Surging Point Absorber Wave Energy Converter, in: Proceedings of the 8th European Wave and Tidal Energy Conference, Uppsala, Sweden, 2009.

[25] L. F. Chen, L. Sun, J. Zang, A. Hillis, Numerical Simulation of Waveinduced Roll of a 2-D Rectangular Barge Using OpenFOAM, in: Proceedings of the 29th International Workshop on Water Waves and Floating Bodies, Osaka, Japan, 2014.

[26] O. Ubbink, Numerical prediction of two fluid systems with sharp interfaces, Ph.D. thesis, Imperial College of Science, Technology and Medicine, 1997. 
[27] H. Rusche, Computational fluid dynamics of dispersed two-phase flows at high phase fractions, Ph.D. thesis, Imperial College of Science, Technology \& Medicine, 2002.

[28] H. Jasak, Error Analysis and Estimation for the Finite Volume Method with Applications to Fluid Flows, Ph.D. thesis, Imperial College of Science,

[29] P. Higuera, J. L. Lara, I. J. Losada, Realistic wave generation and active wave absorption for Navier-Stokes models Aplication to OpenFOAM ${ }^{\circledR}$, Coastal Engineering 71 (2013) 102-118.

[30] OpenCFD, OpenFOAM ${ }^{\circledR}$, website, http://www.openfoam.org/ accessed 29th May 2014, 2014.

[31] N. G. Jacobsen, D. R. Fuhrman, J. Fredsøe, A wave generation toolbox for the open-source CFD library: OpenFOAM ${ }^{\circledR}$, International Journal for Numerical Methods in Fluids 70 (2012) 1073-1088.

[32] N. G. Jacobsen, Contrib/waves2Foam, website, http://openfoamwiki. net/index.php/Contrib/waves2Foam accessed 15th September 2014, 2014 .

[33] M. M. Jakobsen, G. Iglesias, M. Kramer, E. Vidal, Experimental study of forces on point absorber, in: Proceedings of the 5th International Conference Coastlab 14, Varna, Bulgaria, 2014. 